\section{Cell surface proteins Nasrat and Polehole stabilize the Torso-like extracellular determinant in Drosophila oogenesis}

Gerardo Jiménez ${ }^{1,3}$ Acaimo González-Reyes ${ }^{2}$ and Jordi Casanova ${ }^{1}$

${ }^{1}$ Instituto de Biología Molecular de Barcelona (CSIC), 08034 Barcelona, Spain; ${ }^{2}$ Instituto de Parasitología y Biomedicina (CSIC), 18001 Granada, Spain

Structural cell-surface and extracellular-matrix proteins modulate intercellular signaling events during development, but how this is achieved remains largely unknown. Here we identify a novel family of Drosophila proteins, Nasrat and Polehole, that coat the oocyte surface and play two roles: They mediate assembly of the eggshell, and act in the Torso RTK signaling pathway that specifies the terminal regions of the embryo. Nasrat and Polehole are essential for extracellular accumulation of Torso-like, a factor secreted during oogenesis that initiates Torso receptor activation. Stabilization of secreted factors by specialized pericellular proteins may be a general mechanism during signaling and developmental patterning.

Received December 21, 2001; revised version accepted February 15, 2002

Animal cells are surrounded by a complex array of molecules (e.g., components of the extracellular matrix and other secreted proteins) that provide structural support and mediate cell-cell adhesion and communication. Originally considered a passive environment, the cell surface is now viewed as a rich substrate for dynamic interactions influencing cell differentiation, tissue remodeling, and morphogenesis, in both normal and pathological states. For example, recent genetic analyses have revealed dedicated roles of cell-surface proteoglycans in several signaling pathways during development (Bernfield et al. 1999; Perrimon and Bernfield 2000; Selleck 2000). However, little is known about how these functions are exerted and it seems likely that additional cell-surface components and activities remain to be discovered. Here, we identify two novel Drosophila proteins associated to the oocyte surface and investigate their roles in early development.

Drosophila oocytes develop within egg chambers that

[Key Words: Nasrat; Polehole; cell surface; oogenesis; signaling; Drosophila]

${ }^{\text {Sophila] }}{ }^{3}$ Corresponding author.

E-MAIL gjcbmc@cid.csic.es; FAX 34-93-2045904.

Article and publication are at http://www.genesdev.org/cgi/doi/10.1101/ gad.223902. consist of 15 germ-line-derived nurse cells connected to the oocyte, all of them surrounded by a monolayer of somatic follicle cells (Spradling 1993). During oogenesis, the follicle cells secrete structural components of protective shell layers that enclose the egg (Mahowald and Kambysellis 1980; Spradling 1993). In addition, both the nurse and follicle cells provide the oocyte with generic cytoplasmic constituents, plus a number of specific determinants required for early patterning of the embryo. Determinants from the nurse cells (mostly mRNAs) accumulate asymmetrically within the oocyte and are usually activated on fertilization (St Johnston and Nüsslein-Volhard 1992). In contrast, the follicle cells secrete developmental factors that associate to the extracellular surface of the embryo, where they generate signals that activate transmembrane receptors in restricted positions (St Johnston and Nüsslein-Volhard 1992; see below).

Eggshell biogenesis and signaling by extracellular determinants may be interrelated phenomena in Drosophila. Genetic studies have identified two maternal effect loci, $f_{S}(1)$ Nasrat $\left(f_{S}(1) N\right)$ and $f_{s}(1)$ polehole $\left(f_{s}(1) p h\right)$, which are essential for both types of processes (Degelmann et al. 1990, and references therein). Females mutant for most $f_{S}(1) N$ and $f_{S}(1) p h$ alleles lay eggs that collapse after deposition due to abnormalities in the vitelline membrane, the innermost eggshell layer. In addition, the $f_{S}(1) N^{12}$ and $f_{S}(1) p h^{1901}$ mutations (in homozygosis or in heteroallelic combinations with the above alleles) do not affect eggshell formation but cause the loss of the most anterior and posterior (terminal) regions of the embryo (Degelmann et al. 1990). Terminal regions are normally patterned in response to the Torso-like factor secreted by specialized follicle cells at each end of the oocyte (Savant-Bhonsale and Montell 1993; Martin et al. 1994; for review, see Duffy and Perrimon 1994). Torso-like, a protein of unknown biochemical function, is thought to remain associated to the oocyte poles until the first stages of embryogenesis, when it triggers localized processing of the Trunk factor in the perivitelline space between the embryo and the vitelline envelope (Casanova et al. 1995; Casali and Casanova 2001). Cleaved Trunk protein activates the Torso receptor tyrosine kinase present on the embryo surface, which then signals via the Ras/MAPK cascade to induce expression of the tailless (t11) and huckebein $(h k b)$ genes at each pole of the embryo (Duffy and Perrimon 1994; Jiménez et al. 2000). Thus, $f_{S}(1) N$ and $f_{S}(1) p h$ have a dual role in eggshell biosynthesis and terminal cell signaling; however, the nature of the $f_{S}(1) N$ and $f_{S}(1) p h$ products and their function in these processes is unknown.

Here we describe the cloning and characterization of $f_{S}(1) N$ and $f_{S}(1) p h$. We show that these genes encode related leucine-rich proteins that are secreted by the oocyte and remain associated to its plasma membrane. We identify a role of Nasrat and Polehole proteins in crosslinking of vitelline membrane product sV23. In addition, we provide evidence that Nasrat and Polehole are essential for extracellular accumulation of Torso-like at the oocyte surface. Thus, structural cell surface proteins can mediate cell signaling by stabilizing secreted factors in the extracellular space. 


\section{Results and Discussion}

\section{fs $(1) \mathrm{N}$ encodes a putative secreted protein}

$f_{S}(1) N$ has been mapped to chromosomal position 1E-1F (Degelmann et al. 1990). To begin the molecular identification of $f_{S}(1) N$, we searched for new alleles of the gene by mobilizing a collection of P-element insertions in the region (see Materials and Methods). We identified three imprecise excisions of insertion $E P(X) 1336$ that behave as $f_{S}(1) N$ mutations and studied one of them, designated $f_{S}(1) N^{14}$. $f_{S}(1) N^{14}$ behaves as a null allele that affects both eggshell integrity and terminal patterning: females homozygous for $f_{S}(1) N^{14}$ produce collapsed eggs that fail to develop (data not shown), whereas $f_{S}(1) N^{14} / f_{S}(1) N^{12}$ females produce embryos with the terminal phenotype associated with the $f_{S}(1) N^{12}$ allele (Fig. 1A,B; see also Degelmann et al. 1990). Indeed, the latter embryos exhibit severely reduced $t 11$ and $h k b$ expression at the blastoderm stage, similar to the effect of mutations in other components of the terminal pathway (Fig. 1C-F; for review, see Duffy and Perrimon 1994).

The $E P(X) 1336$ insertion is located 200-bp upstream of a novel gene predicted by the Drosophila genome project, CG11411. Molecular analyses showed that $f_{S}(1) N^{14}$ consists in a small deletion $(<700 \mathrm{bp}$ ) of putative promoter and first exon sequences of CG11411 (data not

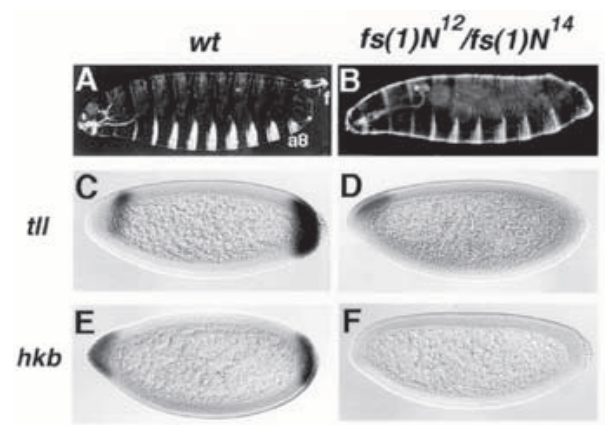

\section{G}

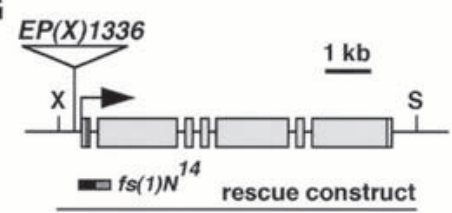

H
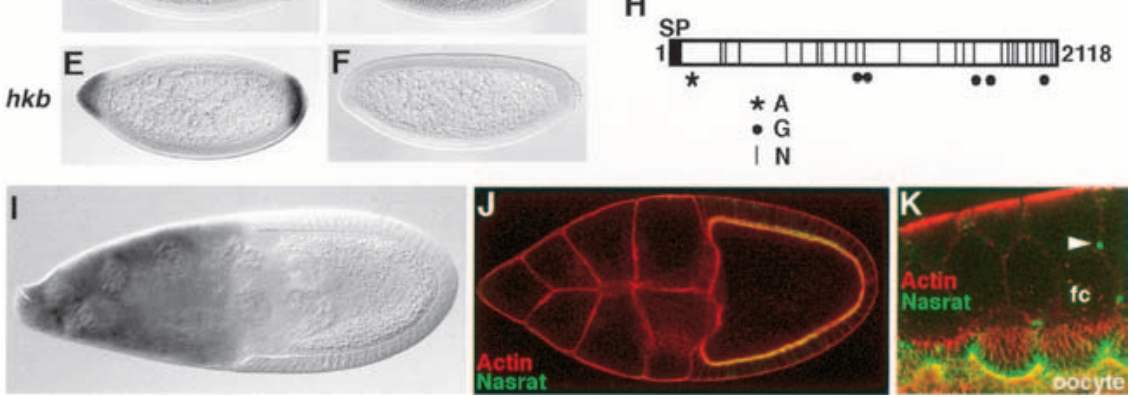

Figure 1. Molecular cloning and characterization of $f_{S}(1) N .(A-F)$ Terminal defects of embryos laid by $f_{S}(1) N^{12} / f_{S}(1) N^{14}$ females. $(A, B)$ Cuticles of embryos derived from wild-type $(A)$ and $f_{S}(1) N^{12} / f_{S}(1) N^{14}(B)$ females; note the lack of terminal structures such as the eighth abdominal segment $(\mathrm{a} 8)$ and filzkörper $(\mathrm{f})$ in the mutant embryo. $(C-F)$ RNA expression patterns of terminal-specific genes $t l l(C, D)$ and $h k b(E, F)$ in embryos produced by wild-type $(C, E)$ and $f_{S}(1) N^{12} / f_{S}(1) N^{14}(D, F)$ females; expression of both genes is strongly reduced or absent in the mutant embryos. $(G)$ Diagram of the $f_{S}(1) N$ locus and the $E P(X) 1336$ insertion. Boxes indicate the position of exons and coding sequences are shaded. The region deleted by imprecise excision $f_{S}(1) N^{14}$ is shown; the hatched segment indicates uncertainty about the precise limit of the deletion. The genomic construct used for transformation rescue is also depicted. (X) XhoI; (S) SpeI. (H) Diagram of the Nasrat protein. (SP) Putative signal peptide at the N terminus; (A) potential ATP/GTP-binding motif [A/G-X(4)-GK-S/T]; (G) putative GAG attachment sites (D/E-X-SG or SG-X-G); (N) N-linked glycosylation motifs (N-X-S/T). (I) fs $(1) N$ RNA expression in the nurse cells at stage 9-10. (J) Stage 10 egg chamber transgenic for the NasratFlu construct stained with anti-Flu antibody (green) and rhodamine-phalloidin to label the cortical actin of the oocyte and follicle cells (red). The tagged protein outlines the periphery of the oocyte. Note the overlap (yellow) of anti-Flu and rhodamine-phalloidin staining at relatively low magnification. $(K)$ Close-up view of the oocyte-follicle cell interface stained as above, showing accumulation of Nasrat on the extracellular side of the oocyte membrane. Note the presence of interconnecting microvilli from both the oocyte and follicle cells (fc). We also see localization of Nasrat-Flu at the ring canals between follicle cells (arrowhead). shown; Fig. 1G). Also, a genomic construct of this gene rescued the phenotypes associated with $f_{S}(1) N$ mutations, demonstrating that CG11411 is $f_{S}(1) N$ (Fig. $\left.1 G\right)$. ing an early embryonic library (see Materials and Methods). The sequence of this clone extends 65-bp upstream dicted exon/intron structure of the gene. $f_{S}(1) N$ encodes (ain 1986). No other putative transmembrane regions were membrane-anchored proteins; there are $25 \mathrm{~N}$-linked cosylation motifs (Hart et al. 1979) and five putative (GAG) attachment sites (Krueger et al. Nasrat accumulates on the oocyte surface

We examined the expression of $f_{S}(1) N$ in ovaries by in situ hybridization. $f_{S}(1) N$ transcripts are detected in the nurse cells throughout most of oogenesis and in early blastoderm embryos, but not in the somatic follicle cells (Fig. 1I; data not shown). This pattern of expression is consistent with a maternal function of $f_{S}(1) N$ in the germline as deduced from genetic analyses (Perrimon and Gans 1983; Degelmann et al. 1990).

Next, we monitored Nasrat protein distribution in the ovary using a transgenic construct encoding a Flutagged Nasrat derivative (Materials and Methods). This construct rescues the sterility of $f_{S}(1) N^{14}$ females completely (data not shown), indicating that the Flu epitope does not interfere with Nasrat function. In early egg chambers (stage 5-6), Nasrat is found in the oocyte cytoplasm (see below). In contrast, by stage 10 the protein localizes to the oocyte periphery (Fig. $1 \mathrm{~J})$. At high magnification, Nasrat is detected apically of cortical actin (visualized with rhodamine-phalloidin; Fig. $1 \mathrm{~J}, \mathrm{~K})$, suggesting that Nasrat lies on the external surface of the oocyte membrane. Moreover, Nasrat shows a finger-like pattern that probably reflects the microvilli formed by the plasma membrane of the oocyte (Fig. $1 \mathrm{~K}$; Mahowald and Kambysellis 1980). We also see actin staining of similar extensions from the plasma membrane of follicle cells, which appear to interdigitate with the oocyte microvilli (Fig. 1K). Finally, there is 
localized accumulation of Nasrat at the ring canals between follicle cells (Fig. 1K). This may result from undetectable $f_{S}(1) N$ expression in those cells, or internalization of Nasrat protein from the extracellular space. Because the primary site of $f_{S}(1) N$ function is the germline (Perrimon and Gans 1983), we have not studied the significance of this localization further.

\section{fs(1)ph encodes a secreted protein related to Nasrat}

As the Drosophila genome project progressed, we noted significant similarities of Nasrat to the CG4790 gene product. The similarity is moderate but extends over a considerable length: $23 \%$ identity in a 700 -amino acid overlap (residues 805-1495 of Nasrat and 430-1119 of CG4790 protein; data not shown). Within this alignment there is a block of 200 amino acids with $25 \%$ identity (Fig. 2A). Because $f_{S}(1) N$ and $f_{S}(1) p h$ share many genetic features (Degelmann et al. 1990), we wondered if CG4790 might be $f_{S}(1) p h$. Consistent with this hypothesis, CG4790 maps to chromosomal position 5C8-10 whereas $f_{S}(1) p h$ lies in the 5C5-D6 interval (Degelmann et al. 1990). We confirmed that CG4790 corresponds to $f_{S}(1) p h$ using a CG4790 transgene that rescued the eggshell and terminal defects caused by $f_{s}(1) p h$ mutations (see Materials and Methods).

A full-length $f_{S}(1) p h$ cDNA clone was obtained from an embryonic library (see Materials and Methods). Its sequence is in agreement with the exon/intron annotations made for CG4790 by the Berkeley Genome Project, except for two differences in splicing sites that make the putative Polehole protein 43 amino acids longer than originally predicted. Like Nasrat, Polehole is rich in leucine residues $(14 \%)$ and contains a putative signal peptide of 25 residues at the $\mathrm{N}$ terminus (von Heijne 1986; Fig. 2B). Polehole also has a large number of potential glycosylation sites, including $26 \mathrm{~N}$-linked glycosylation motifs and two GAG attachment sites (Fig. 2B). Database searches did not detect similarity of Polehole to proteins other than Nasrat, suggesting that these two proteins form a unique family.
Interdependent colocalization of Nasrat and Polehole at the oocyte surface

We analyzed the cellular localization of Polehole in the ovary using an epitope-tag strategy. We generated a Flutagged Polehole construct that rescued the sterility of females homozygous for $f_{S}(1) p h^{1901}$. As in the case of Nasrat, Polehole lies on the outer leaflet of the oocyte and outlines its microvilli (Fig. 2C,D). Thus, Nasrat and Polehole colocalize within a thin layer on the oocyte surface that establishes an intricate pattern of connections with the follicle cells.

To investigate this colocalization further, we assayed accumulation of Flu-tagged Nasrat and Flu-tagged Polehole in $f_{S}(1) p h^{K 646}$ and $f_{S}(1) N^{14}$ mutant ovaries, respectively. In both cases, the tagged derivatives are lost from the oocyte surface (Fig. 2E,F). We do not observe unlocalized Nasrat and Polehole proteins within the mutant oocytes (Fig. 2E,F; data not shown), suggesting a defect in stability rather than transport. These results imply that Nasrat and Polehole are mutually required for their pericellular accumulation, which explains their similar mutant phenotypes. We also examined if this requirement is specific or reflects a general disorganization of the oocyte pericellular environment in the mutant ovaries. To this end, we monitored localization of Nudel, a protein required for dorsoventral patterning that associates to the oocyte surface (Hong and Hashimoto 1995; LeMosy et al. 1998). As shown in Figure 2G, Nudel shows a normal distribution in $f_{S}(1) N^{14}$ egg chambers, arguing that interdependent accumulation of Nasrat and Polehole is selective.

\section{Role of Nasrat and Polehole in eggshell assembly}

The above results suggest that Nasrat and Polehole function coordinately at the oocyte surface. To explore their role in eggshell assembly, we first examined the localization of Nasrat in relation to the nascent vitelline membrane. Double staining for Nasrat and sV23, a vitelline membrane component (Pascucci et al. 1996), showed early cytoplasmic accumulation of Nasrat and

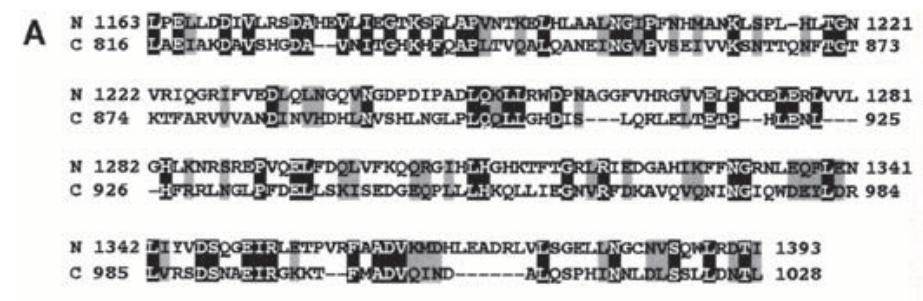

B

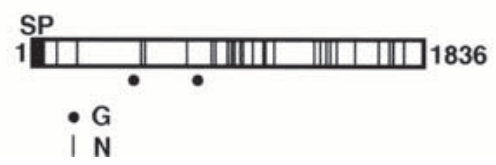

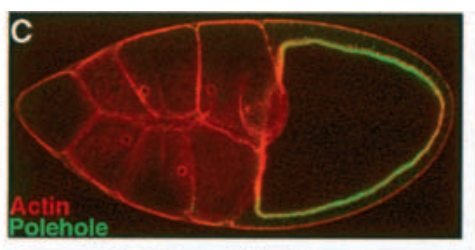
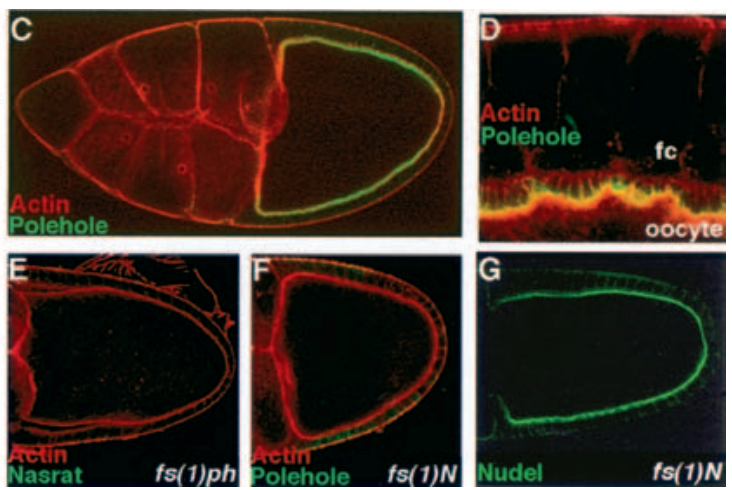

Figure 2. Identification of Polehole and its colocalization with Nasrat at the oocyte periphery. (A) Alignment of Nasrat and CG4790 protein domains showing significant sequence similarity (BLAST E value of 3e-5). Identical and similar residues are shaded in black and gray, respectively. Numbers indicate amino acid positions. (N) Nasrat; (C) CG4790. (B) Diagram of the Polehole protein. (SP) Putative signal peptide; (G) putative GAG attachment sites (D/E-X-SG or SG-X-G); (N) potential N-linked glycosylation motifs (N-X-S/T). (C) Stage 10 egg chamber expressing the Polehole-Flu construct stained with anti-Flu antibody (green) and rhodamine-phalloidin to label cortical actin (red); the tagged protein accumulates on the periphery of the oocyte. $(D)$ Close-up view of the oocyte-follicle cell interface, stained as in $C$. Polehole-Flu outlines the microvilli formed by the oocyte membrane, which associate with similar projections from the follicle cells $(\mathrm{fc})$. (E, $F)$ Loss of Nasrat-Flu $(E)$ and Polehole-Flu $(F)$ staining in $f_{S}(1) p h^{K 646}$ and $f_{S}(1) N^{14}$ mutant ovaries, respectively. $(G) f_{S}(1) N^{14}$ mutant egg chamber stained for Nudel protein; the pattern of accumulation appears completely normal (cf. LeMosy et al. 1998). 

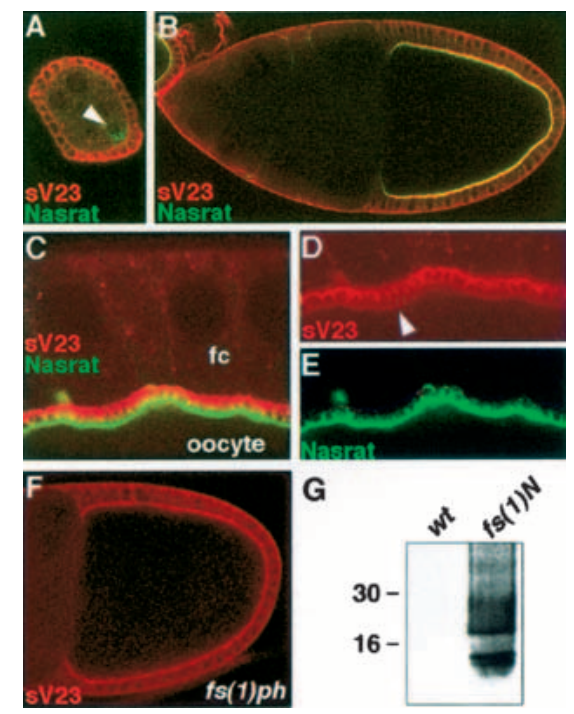

Figure 3. Nasrat and Polehole are required for cross-linking of sV23 product. $(A-E)$ Egg chambers expressing the Nasrat-Flu construct stained with anti-Flu (green) and anti-sV23 (red). (A) Stage 5 egg chamber; Nasrat and sV23 show cytoplasmic accumulation in the oocyte (arrowhead) and follicle cells, respectively. (B) Stage 10 egg chamber; Nasrat and sV23 mark the intercellular space between the oocyte and the follicle cells. $(C-E)$ Close-up view of the stage 10 follicle cortex. Nasrat and sV23 outline the microvilli formed by the oocyte and follicle cells (fc), respectively. We also detect low levels of sV23 on the oocyte surface (arrowhead, D). $(F)$ sV23 staining in a mutant $f_{S}(1) p h^{K 646}$ egg chamber that lacks both Nasrat and Polehole at the oocyte periphery; sV23 appears to accumulate normally. $(G)$ Western blot analysis with sV23 antibody of protein extracts from embryos laid by wild-type and $f_{s}(1) N^{14}$ mutant mothers; note the presence of soluble sV23 species in the vitelline membrane of mutant embryos.

sV23 in the oocyte and the follicle cells, respectively (Fig. 3A; see Materials and Methods). At stage 10, the two proteins marked complementary layers in the space between the oocyte and the follicle cells (Fig. 3B-E). These layers establish a remarkable pattern of connections that appear to reflect the interdigitating microvilli from both cell types (Fig. 3C-E). We also detect weak sV23 staining on the oocyte surface (Fig. 3D). The elaborate interrelation of Nasrat with the vitelline membrane is consistent with a role of this protein in eggshell biogenesis.

Next, we analyzed the distribution of sV23 protein in $f_{S}(1) N^{14}$ and $f_{S}(1) p h^{K 646}$ mutant ovaries, which in both cases simultaneously lack Nasrat and Polehole at the oocyte surface. sV23 remained unaffected in stage 10 egg chambers of both mutant backgrounds (Fig. 3F; data not shown), indicating that sV23 is secreted and begins to accumulate independently of Nasrat and Polehole. This suggests that Nasrat and Polehole mediate subsequent steps of eggshell formation. Such steps include crosslinking modifications of vitelline membrane components that progressively render this membrane insoluble in reducing agents (e.g., DTT) and detergents (LeMosy and Hashimoto 2000, and references therein). It has been shown that Nudel is required for cross-linking of sV23 and $\mathrm{sV} 17$ vitelline membrane proteins via nondisulfide bonds, which are insoluble in DTT (LeMosy and Hashimoto 2000). To test if Nasrat and Polehole also mediate these modifications, blastoderm embryos from either wild-type or $f_{S}(1) N^{14}$ females were extracted with 100
mM DTT and recovery of sV23 protein was assayed by Western blot (see Materials and Methods). Whereas wildtype embryos do not contain soluble sV23 protein, a large amount of product is recovered from embryos laid by $f_{S}(1) N^{14}$ females (Fig. 3G). These results indicate that the combined activities of Nasrat and Polehole are required, directly or indirectly, for nondisulfide cross-linking of sV23 protein during oogenesis.

\section{Role of Nasrat and Polehole in terminal signaling}

The $f_{S}(1) N^{12}$ and $f_{S}(1) p h^{1901}$ alleles do not affect the eggshell but give rise to embryos that lack terminal structures (Degelmann et al. 1990). We identified the molecular lesions associated to $f_{S}(1) N^{12}$ and $f_{S}(1) p h^{1901}$ : P830L and $\mathrm{Y} 742 \mathrm{~N}$, respectively (Fig. 4A). To characterize the $f_{S}(1) N^{12}$ mutation further, we generated a Nasrat derivative carrying a deletion of 161 amino acids encompassing P830 (Nasrat ${ }^{\Delta 161}$; Fig. 4B). Nasrat ${ }^{\Delta 161}$ failed to rescue both the collapsed egg and terminal phenotypes associated with different $f_{S}(1) N$ alleles (data not shown), indicating that it lacks sequences required for both Nasrat functions. In contrast, a deletion of P830 and five adjacent residues (construct Nasrat ${ }^{\Delta 6}$ ) only disrupts terminal patterning (Fig. 4B,C), suggesting that this motif specifically mediates terminal signaling.

What is the role of Nasrat and Polehole in terminal
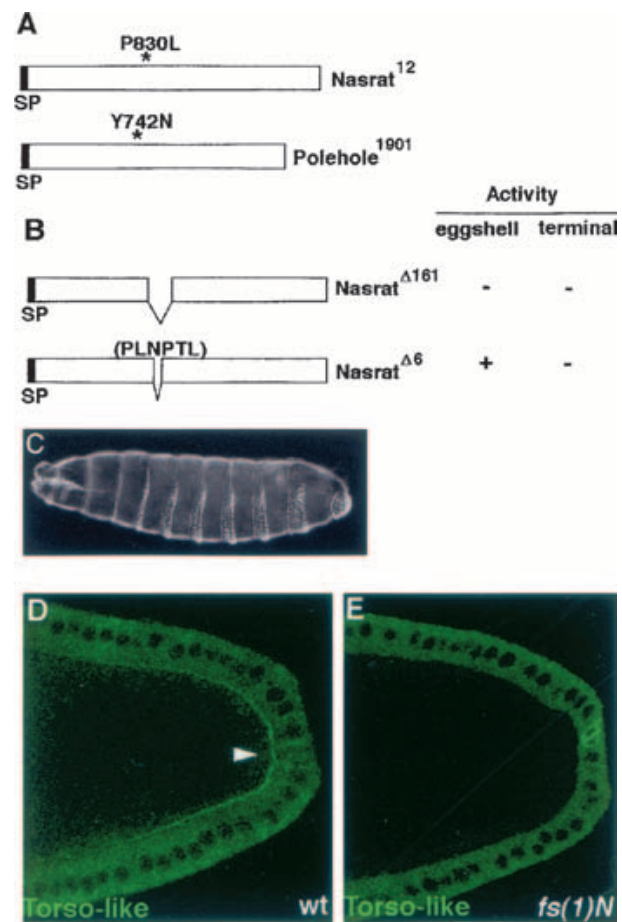

Figure 4. Nasrat and Polehole mediate extracellular accumulation of secreted Torso-like product. (A) Diagram of the molecular lesions associated to the $f_{S}(1) N^{12}$ and $f_{S}(1) p h^{1901}$ mutant alleles; (SP) Signal peptide. $(B)$ Schematic representation of two Nasrat derivatives assayed for rescue of the $f_{S}(1) N^{14}$ null allele. The $\mathrm{Nas}^{\Delta 161}$ protein is inactive with respect to both eggshell and terminal patterning functions, whereas $\mathrm{Nas}^{\Delta 6}$ retains eggshell function but no terminal activity. $(C)$ Embryo laid by a homozygous $f_{S}(1) N^{14}$ female carrying the $\mathrm{Nas}^{\Delta 6}$ construct; note the typical terminal phenotype. $(D, E)$ Stage 10 egg chambers carrying the Torso-like-Flu construct stained with anti-Flu antibody. Note the presence of extracellular Torso-like protein in wild-type (arrowhead, $D)$ but not in $f_{S}(1) N^{14}(E)$ egg chambers. 
signaling? Because Torso-like is the localized determinant for Torso receptor activation, we investigated the effects of loss of Nasrat and Polehole function on Torsolike distribution. Flu-tagged Torso-like protein is detected at stage 10 in the cytoplasm of posterior follicle cells (Fig. 4D; see Materials and Methods). In addition, Torso-like accumulates in a posterior crescent between the follicle cells and the oocyte that probably corresponds to the secreted, active form of the protein (Fig. 4D). This signal forms a gradient that peaks at the posterior end, the site of Torso-like production (SavantBhonsale and Montell 1993; Martin et al. 1994). We first analyzed the effects on Torso-like distribution of the $f_{S}(1) N^{12}$ and $f_{S}(1) p h^{1901}$ mutations that specifically disrupt terminal cell signaling. In both cases, extracellular Torso-like protein is still present between the posterior follicle cells and the oocyte. However, the signal appears consistently weaker than in wild-type ovaries, suggesting that Nasrat and Polehole are required for efficient Torso-like accumulation (data not shown). To test this idea, we examined Torso-like in $f_{S}(1) N^{14}$ ovaries, which lack both Nasrat and Polehole at the oocyte surface. These ovaries showed barely any Torso-like protein between the posterior follicle cells and the oocyte; weak localized staining is still observed in some cases, but most egg chambers lack the extracellular signal (Fig. 4E). These results indicate that Nasrat and Polehole are essential for accumulation and/or stability of secreted Torso-like product.

We also tested if this requirement involves direct physical interactions between Nasrat, Polehole, and Torso-like. Although we observed different interactions, we have been unable to demonstrate their specificity. For example, associations of Nasrat and Polehole with Torso-like were not prevented by the P830L and Y742N mutations (G. Jiménez, unpubl.). It may be difficult to prove relevant associations of Nasrat and/or Polehole with Torso-like if they require specific polysaccharide chains attached to these proteins, as it is thought to occur during binding of cell surface proteoglycans to secreted proteins (e.g., Perrimon and Bernfield 2000).

\section{Conclusion}

Nasrat and Polehole illustrate a role of cell surface molecules as common effectors of eggshell architecture and cell signaling events during development. To mediate these functions, Nasrat and Polehole promote their own accumulation at the oocyte surface, and also stabilize the Torso-like product deposited by follicle cells at each pole of the oocyte. We still do not know how these stabilizations occur molecularly, but one possibility is that Nasrat and Polehole function as protective molecules against unspecific degradation by extracellular proteases present between the oocyte and the follicle cells (e.g., Pascucci et al. 1996). Stabilization of secreted signals by cell surface molecules may be an important mechanism to ensure efficient activation of target receptors in other contexts. Indeed, recent studies have implicated cell surface proteoglycans in signaling by a variety of effectors such as the FGF, Hedgehog, TGF- $\beta$, and Wnt proteins (Bernfield et al. 1999; Perrimon and Bernfield 2000; Selleck 2000), raising the possibility that proteoglycans and related molecules promote accumulation of signaling products in those systems.

\section{Materials and methods}

Fly stocks and crosses

For a description of the $f_{S}(1) N^{12}, f_{S}(1) N^{10}, f_{S}(1) p h^{1901}$, and $f_{S}(1) p h^{K 646}$ alleles (see Degelmann et al. 1990). To identify novel alleles of $f_{S}(1) N, 14$ P-element insertions in the 1E3-1F4 region were mobilized in males and the resulting excisions were tested for complementation of the $f_{s}(1) N^{12}$ allele. Excisions of line $E P(X) 1336$ that failed to complement $f_{S}(1) N^{12}$ were characterized by PCR. Transgenic lines were obtained by injecting the relevant constructs into $y \mathrm{w}$ embryos following standard methods (Spradling 1986). Transgenic constructs were introduced into appropriate mutant backgrounds using standard crossing schemes (details are available on request).

Molecular cloning

A 8.9-kb XhoI-SpeI fragment including the entire $f_{S}(1) N$ transcription unit and $1.4 \mathrm{~kb}$ of $5^{\prime}$ flanking sequences was recovered from cosmid 8D8 (provided by S. Bolshakov, European Drosophila Genome Project). An 8 -kb genomic fragment containing the $f_{S}(1) p h$ transcription unit was generated by PCR from wild-type Oregon flies using the Expand Long Template PCR System (Roche). These genomic fragments rescued the corresponding $f_{S}(1) N$ and $f_{S}(1) p h$ mutations completely. $f_{S}(1) N$ and fs(1)ph cDNA clones were isolated by screening a 0- to 4-h embryonic library kindly provided by N. Brown (University of Cambridge, UK). To ensure the recovery of full-length clones, short $(<1 \mathrm{~kb})$ PCR fragments corresponding to the $5^{\prime}$ region of both genes were used as probes.

DNA sequencing and comparative analyses

All sequences were obtained using automated DNA sequencers (Applied Biosystems). For sequencing of $f_{S}(1) N^{12}$ and $f_{S}(1) p h^{1901}$ alleles, genomic DNA fragments for each mutant gene were amplified by PCR and either subcloned into pBluescript (Stratagene) in duplicate experiments or sequenced directly. Sequences of primers are available on request. Comparative sequence analyses were performed using the BLAST and FASTA programs.

\section{DNA constructs}

All recombinant DNA work was performed according to standard procedures. Coding segments obtained by PCR were sequenced to ensure fidelity during amplification.

To construct Flu-tagged Nasrat, a PCR fragment encoding three tandem copies of the Flu epitope (YPYDVPDYA) was cloned in frame into the unique SfiI site present downstream of the $f_{S}(1) N$ signal peptide sequence. Flu-tagged Polehole was made similarly, by cloning Flu sequences at the equivalent position using a NruI site created in $f_{S}(1) p h$ by PCR-mediated mutagenesis. Finally, Flu-tagged Torso-like was generated by inserting the Flu sequence into a functional torso-like transgene (Savant-Bhonsale and Montell 1993), using the NruI site present downstream of the signal peptide. The final constructs were identical to those employed in the rescue experiments (see above; Savant-Bhonsale and Montell 1993) except that they included Flu sequences.

Nasrat ${ }^{\Delta 161}$ was generated by digesting a plasmid containing the original 8.9-kb $f_{S}(1) N$ genomic fragment with BgIII, thus releasing the sequences to be deleted, and recircularizing it. To generate Nasrat ${ }^{\Delta 6}$, we obtained by PCR-assisted mutagenesis a cDNA fragment carrying the relevant 6-amino-acid internal deletion. This fragment, which included the two BgIII sites present in the $f_{S}(1) N$ sequence, was then digested with BgIII, purified and cloned into the unique BgIII site of construct Nasrat $^{\Delta 161}$.

All constructs made for transformation experiments were assembled in $p C a S p e R 4$ vector. Additional details on the sequence of primers and the construction of plasmids are available on request.

\section{Histochemistry}

Patterns of RNA expression in embryos and ovaries were determined by whole-mount in situ hybridization using digoxygenin-labeled anti-sense RNA probes essentially as described previously (Jiang et al. 1991). For $f_{S}(1) N$ hybridizations, a sense RNA probe was used as a negative control. Signals were detected using a secondary antibody coupled to alkaline phosphatase (Roche). Antibody and rhodamine-phalloidin stainings were performed according to standard procedures; detailed protocols are available on request. In general, we have been unable to monitor protein accumulation in ovaries after stage 10B, possibly owing to progressive 
impermeabilization of the vitelline membrane. Antibodies were used at the following concentrations: monoclonal anti-Flu (clone 12CA5, Roche), 1:100; anti-Nudel $\mathrm{N}$-terminal and C-terminal antibodies (mixed 1:1), 1:500; anti-sV23, 1:500; FITC, Cy2 and Cy3 conjugated secondary antibodies (Jackson Laboratories), 1:200. Confocal images were obtained using a Leica TCS SP2 laser-scanning microscope, and assembled with Adobe Photoshop.

For analysis of sV23 cross-linking, $\sim 50$ nondechorionated eggs $(0-2 \mathrm{~h}$ collection) were homogenized in $100 \mu \mathrm{L}$ of $20 \mathrm{mM}$ Tris- $\mathrm{HCl}(\mathrm{pH} 7.5), 0.15$ $\mathrm{M} \mathrm{NaCl}, 100 \mathrm{mM}$ DTT and boiled for $5 \mathrm{~min}$ to release soluble proteins. Samples were centrifuged to remove insoluble material and supernatants were analyzed by SDS-PAGE followed by Western immunoblotting with sV23 antibody at 1:800 dilution.

\section{Acknowledgments}

We thank M. Furriols, S. González-Crespo, E. Sánchez-Herrero, and G. Struhl for critical comments on the manuscript, and C. Caelles, C. Hashimoto, E. LeMosy, G. Struhl, G. Waring, and the European Drosophila Genome Project for reagents. G.J. was supported by a contract from the Spanish Ministerio de Educación y Cultura (MEC). This work was funded by the Spanish MEC and the Generalitat de Catalunya (C.R. Biotecnologia).

The publication costs of this article were defrayed in part by payment of page charges. This article must therefore be hereby marked "advertisement" in accordance with 18 USC section 1734 solely to indicate this fact.

\section{References}

Bernfield, M., Götte, M., Park, P.W., Reizes O., Fitzgerald M.L., Lincecum, J., and Zako, M. 1999. Functions of cell surface heparan sulfate proteoglycans. Annu. Rev. Biochem. 68: 729-777.

Casali, A. and Casanova, J. 2001. The spatial control of Torso RTK activation: A C-terminal fragment of the Trunk protein acts as a signal for Torso receptor in the Drosophila embryo. Development 128: $1709-1715$

Casanova, J., Furriols, M., McCormick, C.A., and Struhl, G. 1995. Similarities between trunk and spätzle, putative extracellular ligands specifying body pattern in Drosophila. Genes \& Dev. 9: 2539-2544.

Degelmann, A., Hardy, P.A., and Mahowald, A.P. 1990. Genetic analysis of two female-sterile loci affecting eggshell integrity and embryonic pattern formation in Drosophila melanogaster. Genetics 126: 427434.

Duffy, J.B. and Perrimon, N. 1994. The Torso pathway in Drosophila: Lessons on receptor tyrosine kinase signaling and pattern formation. Dev. Biol. 166: 380-395.

Hart, G.W., Brew, K., Grant, G.A., Bradshaw, R.A., and Lennarz, W.J. 1979. Primary structural requirements for the enzymatic formation of the N-glycosidic bond in glycoproteins. J. Biol. Chem. 254: 97479753.

Hong, C.C. and Hashimoto, C. 1995. An unusual mosaic protein with a protease domain, encoded by the nudel gene, is involved in defining embryonic dorsoventral polarity in Drosophila. Cell 82: 785-794.

Jiang, J., Kosman, D., Ip, Y.T., and Levine, M. 1991. The dorsal morphogen gradient regulates the mesoderm determinant twist in early Drosophila embryos. Genes \& Dev. 5: 1881-1891.

Jiménez, G., Guichet, A., Ephrussi, A., and Casanova, J. 2000. Relief of gene repression by Torso RTK signaling: role of capicua in Drosophila terminal and dorsoventral patterning. Genes \& Dev. 14: 224 231.

Krueger, R.C., Fields, T.A., Hildreth IV, J., and Schwartz, N.B. 1990. Chick cartilage chondroitin sulfate proteoglycan core protein. J. Biol. Chem. 265: 12075-12087.

LeMosy, E.K. and Hashimoto, C. 2000. The Nudel protease of Drosophila is required for eggshell biogenesis in addition to embryonic patterning. Dev. Biol. 217: 352-361.

LeMosy, E.K., Kemler, D., and Hashimoto, C. 1998. Role of Nudel protease activation in triggering dorsoventral polarization of the Drosophila embryo. Development 125: 4045-4053.

Mahowald, A.P. and Kambysellis, M.P. 1980. Oogenesis. In The genetics and biology of Drosophila (ed. M. Ashburner and T.R.F. Wright), pp. 141-224. Academic Press, New York, NY.
Martin, J.-R., Raibaud, A., and Ollo, R. 1994. Terminal pattern elements in Drosophila embryo induced by the torso-like protein. Nature 367: 741-745.

Pascucci, T., Perrino, J., Mahowald, A.P., and Waring, G.L. 1996. Eggshell assembly in Drosophila: Processing and localization of vitelline membrane and chorion proteins. Dev. Biol. 117: 590-598.

Perrimon, N. and Gans, M. 1983. Clonal analysis of the tissue specificity of recessive female-sterile mutations of Drosophila melanogaster using a dominant female-sterile mutation $f_{s}(1) K 1237$. Dev. Biol. 100: 365-373.

Perrimon, N. and Bernfield, M. 2000. Specificities of heparan sulphate proteoglycans in developmental processes. Nature 404: 725-728.

Saraste, M., Sibbald, P.R., and Wittinghofer, A. 1990. The P-loop-A common motif in ATP- and GTP-binding proteins. Trends Biochem. Sci. 15: 430-434.

Savant-Bhonsale, S. and Montell, D.J. 1993. torso-like encodes the localized determinant of Drosophila terminal pattern formation. Genes \& Dev. 7: 2548-2555.

Selleck, S.B. 2000. Proteoglycans and pattern formation: Sugar biochemistry meets developmental genetics. Trends Genet. 16: 206-212.

Spradling, A.C. 1986. P element-mediated transformation. In Drosophila: A practical approach (ed. D.B. Roberts), pp. 175-197. IRL Press, Oxford, UK.

. 1993. Developmental genetics of oogenesis. In The development of Drosophila melanogaster (ed. M. Bate and A. Martínez Arias), pp. 1-70. Cold Spring Harbor Laboratory Press, Cold Spring Harbor, NY.

St Johnston, D. and Nüsslein-Volhard, C. 1992. The origin of pattern and polarity in the Drosophila embryo. Cell 68: 201-219.

von Heijne, G. 1986. A new method for predicting signal sequence cleavage sites. Nucleic Acids Res. 14: 4683-4690. 


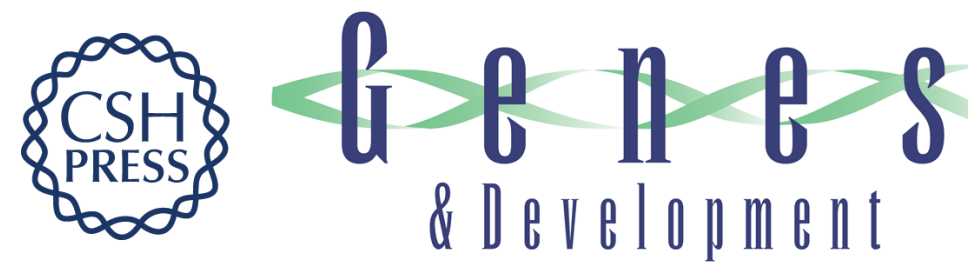

\section{Cell surface proteins Nasrat and Polehole stabilize the Torso-like extracellular determinant in Drosophila oogenesis}

Gerardo Jiménez, Acaimo González-Reyes and Jordi Casanova

Genes Dev. 2002, 16:

Access the most recent version at doi:10.1101/gad.223902

Related Content Locking the Signal at the Poles

Sci. STKE April, 2002 2002: tw152

References This article cites 20 articles, 9 of which can be accessed free at: http://genesdev.cshlp.org/content/16/8/913.full.html\#ref-list-1

Articles cited in:

http://genesdev.cshlp.org/content/16/8/913.full.html\#related-urls

License

Email Alerting Receive free email alerts when new articles cite this article - sign up in the box at the top right corner Service of the article or click here.

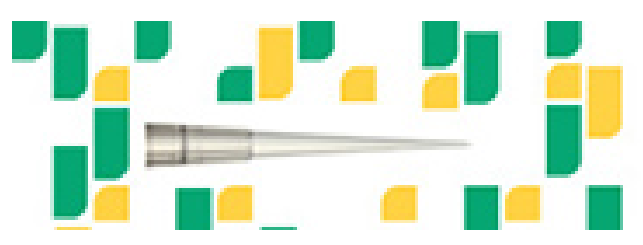

